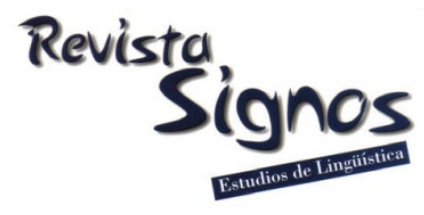

\title{
Estudio de variación en el uso de atenuación I: Hacia una descripción de patrones dialectales y sociolectales de la atenuación en español*
}

\author{
$V$ ariationist study of mitigation I: Towards a description of \\ dialectal and sociolectal patterns of mitigation in Spanish
}

\section{Ana María Cestero Mancera}

UNIVERSIDAD De ALCALÁ

ESPAÑA

anam.cestero@uah.es

\author{
Marta Albelda Marco \\ UNIVERSITAT DE VALÈNCIA \\ ESPAÑA \\ marta.albelda@uv.es
}

Recibido: 17-I-2020 / Aceptado: 21-VII-2020

DOI: $10.4067 /$ S0718-09342020000300935

\section{Resumen}

La atenuación es un fenómeno pragmático de gran complejidad que se ha convertido en objeto de estudio priorizado en las últimas décadas, en las que se ha abordado, especialmente, desde disciplinas que trabajan con el habla, como la pragmática y el análisis del discurso. Uno de los aspectos menos trabajado en relación con las estrategias de atenuación en español, no obstante, es la variabilidad sociopragmática y geolectal en usos y recursos de atenuación. Este último es el cometido fundamental de este trabajo -que se complementa con la investigación presentada en el artículo de Albelda y Cestero (en este mismo volumen, 2020)-, en el que se pretende dar cuenta del estado actual de la investigación variacionista sobre la estrategia atenuadora, especialmente desarrollada en el marco del Proyecto para el estudio sociolingüístico del español de España y América (PRESEEA). En este artículo, se presentan el marco teórico y metodológico del estudio sociogeolectal de la atenuación y los datos relevantes obtenidos de la comparación y el análisis de lo que acontece en el habla de comunidades urbanas estudiadas, como Madrid, Valencia, Las Palmas de Gran Canaria, Santiago de Chile (Chile) y Puebla (México), entre otras, con respecto a la frecuencia de producción de atenuación, a la función para la que se emplea en interacción transaccional, así como a los recursos más productivos en cada comunidad. Todo ello permite vislumbrar convergencias y divergencias, y apuntar patrones dialectales y sociolectales.

Palabras Clave: Atenuación, patrones sociopragmáticos, variación geolectal, PRESEEA, español hablado. 


\begin{abstract}
The study of mitigation, a complex pragmatic phenomenon, has been prioritized in recent decades. It has been approached through linguistic disciplines related to speech, especially pragmatics and discourse analysis. Nevertheless, the sociopragmatic and geolectal variability of mitigation in Spanish has barely been studied. This is the main objective of this paper (which complements the research presented in Authors, this volume), which aims to examine the state of the art on sociolinguistic variation in mitigation strategies, especially within the framework of the Project for the Sociolinguistic Study of Spanish in Spain and America (PRESEEA). The present paper includes the theoretical framework and methodology for the sociodialectal study of mitigation and summarises the main data obtained from a comparison of mitigation in five urban geographic communities: Madrid, Valencia, Las Palmas, Santiago (Chile), and Puebla (Mexico). The comparison addresses the frequency of mitigation, its different functions and the most productive resources in each community. The findings of the present report show convergences and divergences and draw a picture of dialectal and sociolectal patterns in mitigation.
\end{abstract}

Key Words: Mitigation, sociopragmatics patterns, dialectal variation, PRESEEA, spoken Spanish.

\title{
INTRODUCCIÓN
}

Los primeros estudios de la atenuación pragmática desde una perspectiva variacionista en español cumplen ahora diez años; sin embargo, a pesar de tratarse de un intervalo corto de tiempo, la atención recibida ha producido un considerable avance en el conocimiento del funcionamiento de la estrategia. Uno de los principales impulsores de esta línea de estudios para el español ha sido el Proyecto para el estudio sociolingüistico del español de España y América (PRESEEA), que se ha dedicado particularmente al análisis de la variación sociolectal y dialectal de diversos elementos y fenómenos de la lengua española hablada, de todos los niveles lingüísticos (Moreno Fernández, 1996; Cestero, 2012a; Moreno Fernández \& Cestero, en prensa). En el año 2010, PRESEEA comienza su andadura en el estudio de fenómenos pragmáticos, con la elección de la atenuación. Tras la realización de varios estudios piloto sobre el funcionamiento de la atenuación en el habla de Madrid y de Valencia (Albelda, 2011, 2012; Albelda \& Cestero, 2011; Cestero, 2011a, 2011b; Cestero, 2012a, 2012b), se elabora una primera ficha para el análisis de la categoría pragmática en los corpus sociolingüísticos del español. Posteriormente, trabajos más completos de Albelda y Cestero (Albelda, 2013, 2018; Cestero, 2012b, 2015, 2017) aplican y validan la ficha, y, una vez considerada apta para ser compartida, la ponen a disposición de otros investigadores con el fin de impulsar el estudio sociopragmático y dialectal de la atenuación. La acogida fue muy buena y, hoy en día, como se muestra en este artículo, están plenamente incorporados al estudio coordinado de la atenuación los equipos PRESEEA de Las Palmas de Gran Canaria, Granada, Sevilla, Santander, Barranquilla, La Habana, Puebla, Monterrey y Santiago de Chile, entre otros. 
El objetivo del trabajo que aquí introducimos es dar cuenta del momento actual en el estudio sociolingüístico y geolectal de la atenuación tras diez años de su inicio coordinado y sistemático en el marco del PRESEEA, lo que permite, a su vez, establecer el estado de la cuestión a partir de un rápido trazado de su recorrido. Para ello, hemos articulado las acciones en dos procesos principales: (i) compilar y ordenar los análisis de la atenuación llevados a cabo desde la sociolingüística sobre corpus de lengua hablada del español, en concreto, de entrevistas semidirigidas de registro medio de distintas urbes hispanohablantes; en el artículo de Albelda y Cestero que complementa a este en el presente volumen, se atiende también a trabajos en los que se compara PRESEEA con otros corpus, y (ii) obtener una primera descripción de los patrones sociolectales y dialectales de la atenuación, mediante la comparación y el análisis de los datos y hallazgos de las investigaciones realizadas y revisadas.

Como 'diez años no son nada', nos encontramos en pleno avance en el estudio sociolingüístico y dialectal de la atenuación, por eso únicamente podemos hablar de 'hacia la descripción de patrones'. Ahora bien, el desarrollo es cada vez mayor y, además, en él, cobra un protagonismo cada vez más importante no solo el conocimiento del funcionamiento de la variación diatópica y diastrática, sino, también de la diafásica. En relación con ello, creemos oportuno mencionar que, en los últimos años, al estudio sobre los corpus PRESEEA se ha unido la investigación sobre otros corpus recogidos con control de variables sociales y dialectales, especialmente de conversaciones coloquiales (Briz, 2007, 2012; Briz \& Albelda, 2013). Tenemos, así, entrevistas semidirigidas transaccionales y conversaciones coloquiales de distintas urbes españolas e hispanoamericanas, en registro medio e informal (de los corpus Val.Es.Co. y Ameresco, Briz \& Grupo Val.Es.Co. (2002), Albelda \& Estellés, en línea), lo que, sin duda, permitirá establecer patrones con mayor propiedad y dar cuenta de los movimientos de convergencia o divergencia entre variedades sociogeolectales, así como de valores identitarios y de proyección de identidad o de estrategias comunicativas interaccionales. Aunque aún son muy pocos los trabajos en los que se comparan datos obtenidos del estudio de estos dos corpus (y de otros), hemos querido también dar cuenta de ellos en este artículo.

Ofrecemos, a continuación, la información a la que acabamos de aludir, en dos artículos en este volumen, que podrían tratarse también de forma independiente. Comenzamos con este primer trabajo en el que, tras la revisión de los últimos presupuestos teóricos en la definición de la atenuación y del avance en su estudio desde la sociopragmática (apartado 1, marco teórico), se presenta la metodología de análisis del fenómeno desarrollada en el marco del proyecto PRESEEA (apartado 2) y que sirve de eje estructurador del estado de la cuestión. En el apartado 3, se exponen, organizados $\mathrm{y}$, cuando ha sido posible, comparados, los resultados de estudios completos variacionistas sobre el fenómeno pragmático que nos ocupa, lo que nos 
permite, como se verá, establecer patrones sociolectales y dialectales en el uso y funcionamiento de la estrategia interaccional.

\section{La atenuación desde la sociopragmática}

La investigación llevada a cabo hasta la fecha ha mostrado que la atenuación constituye un verdadero indicador de variación diatópica, diastrática y diafásica -en situaciones y registros, y en géneros discursivos. El presente trabajo pretende organizar y analizar, por partes y en su conjunto, los resultados a los que hemos podido acceder sobre los tres tipos de variación, especialmente la sociolectal y la dialectal, para establecer, a partir de ellos, patrones de su uso y funcionamiento, y comenzar la elaboración de un mapa de la atenuación en español.

La atenuación, en tanto que es una estrategia retórica y social, se calcula y formula materialmente en cada situación comunicativa particular; es, por tanto, un fenómeno de naturaleza pragmática. Ahora bien, los culturemas lingüísticos y comunicativos de la zona geográfica de donde provienen los hablantes, así como su caracterización social, influyen en la elección de su forma, frecuencia y función. Este tipo de influencias, en general, no se han tenido en cuenta en los estudios desarrollados, al centrarse casi exclusivamente en la elaboración de una definición pragmáticolingüística del fenómeno para acotar los usos lingüísticos que la cumplen. Sin embargo, como han mostrado las investigaciones sobre atenuación en el marco del PRESEEA, si se quiere conocer en profundidad el fenómeno y llegar a su caracterización completa, es necesario estudiarlo en corpus discursivos y analizar, junto a su caracterización lingüística, factores contextuales, cuya influencia es determinante, esto es, factores sociales, culturales y situacionales.

Las primeras aproximaciones a la atenuación en lingüística (esto es, en la lingüística como ciencia, sin tener en cuenta su consideración como recurso en la retórica clásica) señalan su vinculación con el lenguaje difuso, vago o aproximado, en la expresión semántica proposicional (Lakoff, 1972; Overstreet, 2011), así como su papel minimizador del grado de fuerza ilocutiva de los enunciados (Fraser, 1980; Holmes, 1984; Bazzanella, Caffi \& Sbisá, 1991; Briz, 1995, 2003; Caffi, 1999, 2007; Sbisà, 2001; Thaler, 2012). También, comúnmente, se han establecido conexiones estrechas entre la atenuación lingüística con la cortesía verbal (Briz, 2007, 2012; Schneider, 2013, 2017), lo que indica que estamos ante una herramienta con función social en la lengua. Siendo Lakoff (1972) el primer trabajo lingüístico sobre la atenuación, desde entonces hasta hoy, la caracterización de la atenuación se ha ido abriendo, de manera determinante, hacia lo extralingüístico.

Con el avance en la investigación de este fenómeno, se ha hecho necesario identificar los rasgos de cada expresión lingüística para ejercer una función atenuante en un determinado contexto. Los diversos estudios sobre el tema han ido destacando rasgos identificativos, aunque sin ser coincidentes en todas las definiciones; asimismo, 
apenas ha habido interés por buscar una definición unitaria y exhaustiva. Muestra de ello es la tipologización de rasgos que se ofrece a continuación, extraída de la bibliografía accesible, con la mención de los autores que respaldan las diferentes características:

i. reducción de la precisión (vaguedad) y/o de la cantidad en el contenido proposicional de lo dicho (Lakoff, 1972; Prince, Bosk \& Frader, 1982; Channell, 1994; Briz, 1995; Caffi, 1999, 2007; Cutting, 2007, 2015; Myre 2009; Mihatsch, 2012; Voghera 2013; Llopis, 2016);

ii. reducción escalar, reducción del grado de fuerza ilocutiva del enunciado (Holmes, 1984; Bazzanella et al., 1991; Briz 1995; Caffi, 1999; Sbisà, 2001, Thaler, 2012);

iii. reducción del compromiso del hablante con lo dicho y/o con las obligaciones respecto a sus interlocutores, reducción de la certeza epistémica o de la obligación deóntica (Briz, 1995, 2007; Martinovski, 2006; Czerwionka, 2012; Schneider, 2013, 2017), y

iv. reducción del impacto de los efectos no deseados en las imágenes de los interlocutores (Fraser, 1980; Briz, 2007; Czerwionka, 2012; Thaler, 2012; Hernández, 2013; Schneider, 2013; Albelda 2016; Cestero, en prensa).

Del primero al último de los cuatro rasgos anteriores se aprecia una gradación, desde rasgos más semánticos a más pragmáticos y sociales. Asimismo, se puede observar que, al definir la atenuación, no todos los autores consideran el rasgo de la repercusión sobre la imagen. Sin embargo, de acuerdo con Albelda (2016), existen dos tipos de evidencias que dan cuenta de la necesidad de contar con la incidencia de la imagen como rasgo necesario y fundamental (aunque no suficiente) en la definición de la atenuación:

(a) la falta de operatividad de las definiciones que no incluyen la incidencia de la imagen (iv); el resto de criterios (i-iii) habitualmente no permite al analista el reconocimiento pleno de la atenuación, puesto que no son obligatorios, es decir, no siempre se dan todos en un elemento que se considera atenuado. Según los casos, un uso atenuante responderá a uno o varios de ellos ${ }^{1}$.

(b) la revisión de diversas investigaciones hechas sobre datos en las que se analizan atenuantes en corpus de diversos géneros y en las que las formas reconocidas como atenuantes por parte del investigador incluyen siempre la prevención o reparación de problemas de imagen, sea algo consciente o inconsciente por parte del analista. 
Teniendo en cuenta todo lo anterior, y habiendo seguido la trayectoria desarrollada y los avances logrados en el estudio teórico-metodológico de la atenuación, consideramos que la siguiente definición -que aúna las de Albelda (2016) y Albelda y Briz (2020) - puede ser la más completa y unitaria para dar cuenta del fenómeno estudiado:

"La atenuación es una estrategia retórico-pragmática originada por necesidades de protección de la imagen (propia o ajena) ${ }^{2}$, para proteger, suavizar y reparar los posibles efectos perjudiciales para el adecuado desarrollo de la comunicación. Se expresa a través de mecanismos de lenguaje vago que difuminan el contenido proposicional, de minimización de la cantidad o cualidad semánticas o directamente reduciendo la fuerza ilocutiva de los actos de habla y formulando un menor compromiso con lo dicho. Genera una implicatura conversacional a través de la indireccionalidad en la expresión de la verdadera intención del emisor".

Como señala la definición, la protección de la imagen es el origen de la atenuación, lo que la motiva y provoca su realización. Los estudios realizados sobre muestras de habla de los corpus PRESEEA han derivado en una mayor precisión de la caracterización de la atenuación. A tal respecto, conviene mencionar el grado de incidencia de la imagen en el empleo de la atenuación. Cestero (en prensa) propone un continuum en el tipo de mecanismos atenuadores en función de la mayor o menor exposición de la imagen propia: en un polo, están los mecanismos atenuantes que hacen visible la implicación de la figura del hablante (como la corrección de lo que se dice o se hace), mientras que, en el polo opuesto, están los que distancian más al hablante y, por tanto, exponen en menor grado la imagen (por ejemplo, la desfocalización e impersonalización). En el siguiente apartado se presenta con mayor detalle la nómina de mecanismos y recursos de atenuación estudiados desde la sociolingüística.

\section{Metodología para el estudio sociolingüístico de la atenuación}

\subsection{La entrevista sociolingüística y el diseño de los corpus orales PRESEEA}

Uno de los objetivos principales del PRESEE $A$ es reunir un gran corpus de lengua española hablada con una serie de parámetros comunes, de manera que sean fácilmente comparables las investigaciones sobre sus muestras. Se aplica una misma metodología sociolingüística en una amplia red de ciudades de Hispanoamérica y de España (Moreno Fernández, 1996; Cestero, 2012b; Moreno Fernández \& Cestero, en prensa). Para recoger las muestras de habla se parte de una estratificación de la población en función de tres factores sociales: sexo, edad y nivel de estudios. Se realizan entrevistas semidirigidas a partir de módulos temáticos que permiten 
homogeneidad en el tratamiento de temas (el tiempo, lugar donde se vive, familia y amistad, costumbres, peligro de muerte, anécdotas importantes en la vida, deseo de mejora económica) y en la recogida de distintas secuencias textuales (expositivas, narrativas, descriptivas, argumentativas, etc.), de las que solo se analizan las intervenciones del informante ${ }^{3}$. Se trata, por tanto, de un registro semiformal de lengua, en una situación comunicativa de distancia intermedia (solidaridad reducida) y escasa inmediatez comunicativa, que, en principio, de acuerdo con Briz (2007), está correlacionada con una mayor presencia de atenuantes.

En la actualidad, son muchos ya los corpus PRESEEA finalizados (Moreno Fernández \& Cestero, en prensa). Cada uno de ellos está compuesto por entre 54 y 108 entrevistas, dependiendo de la población de la ciudad, con una duración media de 50 minutos cada una. En las muestras, los informantes son seleccionados cubriendo cuotas fijas; así, en cada corpus, la mitad de las entrevistas son de mujeres y la mitad, de hombres, de tres grupos etarios (jóvenes, de 20 a 34 años, adultos, de 35 a 54 años, y mayores, de 55 años o más) y de tres niveles de instrucción (educación básica o primaria, media o secundaria y alta o universitaria).

De los equipos que disponen de corpus acabados (grabados, transcritos y etiquetados), se han analizado hasta hoy para el estudio de la atenuación los que recoge la siguiente tabla 1 . Sin embargo, solo algunos de ellos -como se aprecia en la tabla- han llevado a cabo el estudio sociopragmático completo:

Tabla 1. Estudio sociolingüístico de la atenuación: Geolectos.

\begin{tabular}{|l|l|l|}
\cline { 2 - 3 } \multicolumn{1}{c|}{} & \multicolumn{1}{c|}{$\begin{array}{c}\text { Estudios completos } \\
\text { de la atenuación }\end{array}$} & \multicolumn{1}{|c|}{$\begin{array}{c}\text { Estudios en profundidad } \\
\text { de secuencias, actos de habla o } \\
\text { recursos de atenuación }\end{array}$} \\
\hline \multirow{4}{*}{ Hispanoamérica } & Santiago de Chile (Chile) & Monterrey (México) \\
\cline { 2 - 3 } & Puebla de Zaragoza (México) & Barranquilla (Colombia) \\
\cline { 2 - 3 } & & Medellín (Colombia) \\
\cline { 2 - 3 } & & La Habana (Cuba) \\
\cline { 2 - 3 } & & Caracas (Venezuela) \\
\cline { 2 - 3 } España & Mérida (Venezuela) \\
\hline & Madrid & Santiago de Chile (Chile) \\
\cline { 2 - 3 } & Valencia & Madrid \\
\cline { 2 - 3 } & Las Palmas de Gran Canaria & Valencia \\
\cline { 2 - 3 } & Granada & Sevilla \\
\hline
\end{tabular}

Disponemos, hasta ahora, de resultados prácticamente completos sobre el uso, el funcionamiento y la producción de atenuación en cinco grandes urbes: Madrid, Valencia, Las Palmas de Gran Canaria, Santiago de Chile y Puebla de Zaragoza; se está concluyendo el estudio de Granada, pero aún no tenemos datos concretos. Sobre los hallazgos de estos cinco geolectos se establecerá una aproximación a patrones sociopragmáticos y dialectales del fenómeno interaccional, que, a su vez, servirá de 
modelo, guía y punto de comparación para describir patrones en otras zonas, a partir de resultados parciales obtenidos en estudios en profundidad de determinadas secuencias discursivas, actos de habla de distinto tipo o algunos recursos concretos de atenuación.

\subsection{Ficha de variables y variantes para análisis sociopragmático de la atenuación}

La atenuación es un fenómeno comunicativo que no está codificado lingüísticamente, de manera que su identificación es contextual. En ese sentido, y como se ha dicho, para el reconocimiento de la atenuación en los corpus, además de la definición de atenuación manejada (sección 1.), es necesario contar con otro tipo de factores pragmático-situacionales y sociales que pueden afectar a su uso. La ficha para el estudio de la atenuación fue realizada inicialmente de forma piloto por Cestero y Albelda. Tras validarla, se fue afinando a partir de los estudios que iban efectuándose, hasta quedar revalidada en 2014, con una extensión fundamental que permitía el estudio completo y profundo de la atenuación en cualquier tipo de actividad interaccional y discursiva (Albelda, Briz, Cestero, Kotwica \& Villalba, 2014; Cestero, 2014, Cestero \& Rodríguez, 2014). La ficha general establecida recoge, como variable dependiente, la función de la atenuación, y, como variables independientes, las que se mostraron implicadas en la estrategia atenuadora en las investigaciones realizadas, con sus variantes concretas, que pueden agruparse en cuatro grupos: procedimiento de atenuación, factores estructurales, factores enunciativos y factores situacionales, sociales y geográficos.

(A) Función del atenuante en el discurso. Se han propuesto diversas tipologías de función según los estudios, aunque la mayor parte ha optado por una división general de tres funciones de la atenuación relacionadas con la repercusión de la imagen y con el momento de la acción atenuadora, previo o posterior al daño o riesgo que puede sufrir la imagen: autoprotección, prevención o reparación (Briz \& Albelda, 2013; Albelda et al., 2014).

(B) Mecanismos lingüísticos (y paralingüísticos) para atenuar. En el estudio coordinado de PRESEEA, se estableció una nómina inicial de 25 mecanismos, a los que se añadió una variable abierta para incluir otros no previstos entre los más frecuentes. En un estudio posterior (Albelda \& Cestero, 2011), se agruparon los recursos en nueve grupos más amplios, reducidos después a siete (Cestero, en prensa), en función de los movimientos comunicativos y estratégicos generales que se activan en la negociación y que abordan la comunicación desde la (in)acción del yo-hablante (categorías 1 y 2), desde el propio contenido comunicativo (categorías 3, 4 y 5) y desde la (in)acción del tú-interlocutor (categorías 6 y 7). Bajo estos movimientos generales se pueden subsumir los mecanismos lingüísticos específicos de atenuación identificados hasta ahora ${ }^{4}$. Los siete grupos, a su vez, se gradúan en una escala que va 
de un mayor a un menor involucramiento de la participación del hablante y en la que su imagen se expone cada vez menos; así, de las categorías 1 a la 7 vemos cómo se desenfoca cada vez más al hablante como actor de la acción comunicativa y de la acción atenuadora (Cestero, en prensa):

1. Recursos que corrigen o reparan lo dicho o hecho:

- Expresiones de disculpa

- Movimientos de corrección o reformulación con o sin marcadores discursivos

- Elementos prosódicos, paralingüísticos o gestuales

(1)

E.: o sea tú crees por ejemplo la- laa los inmigrantes que procedían de la misma provincia o de zonas próximas tendían aa a vivir cerca

I.: sí// y a ayudarse/ sí/ y de hechoo // ha sido- sigue siendo así aunque see está perdiendoo cada vez esa tendencia aa/ < vacilación/> aa/ a ayudarse y a ser más/ $<$ vacilación/> una piña yy más// aatender aa solucionaar o ayudarse en cuantoo que seaa posible

(MADR-VAL_M23_035)

2. Recursos que acotan o restringen lo que se dice o se hace:

- Construcciones acotadoras de la opinión a la propia persona o a un ámbito

- Concesividad

- Estructuras sintácticas que restringen el acto de habla, el alcance de lo dicho, mediante estructuras condicionales, concesivas o temporales.

(2)

E.: ¿๐ algo? / ¿alguna vez que te hayas asustado? ¿te han robado alguna vez?

I.: yo de eso nada

E.: ¿nunca? / ¿tú crees que este barrio es peligroso? //

I.: para mí / no / para mí

E.: ¿ha habido? ¿nunca te has mezclado en nada

I.: no nunca

(MADR-VAL_M11_005)

3. Recursos que rebajan lo que se dice o se hace

- Verbos, construcciones verbales y partículas discursivas modales que expresan opinión en forma de duda o de probabilidad

- Verbos, construcciones verbales y partículas discursivas que expresan fingimiento de incertidumbre, de incompetencia o de ignorancia

- Usos modalizados de los tiempos verbales

- Peticiones, preguntas, mandatos u órdenes expresadas de forma indirecta

(3)

E.: ¿has oído tú que haya pasado algo grave realmente en Vallecas dee- en relación con delincuencia o algo? 
I.: jhombre! pues tenemos estos// pues yo no sé/ hombre hay gentee a lo mejor más cercaa estamos más cerca de barrios de droga/ y esos ajustes de cuenta que dicen de vez en cuando// bueno pues/ pero también lo hay en Carabanchel y lo habrá en Villaverde Alto o en// no sé

(MADR-VAL_M33_053)

4. Recursos que minimizan o difuminan la cantidad o cualidad de lo que se dice

- Modificadores morfológicos internos: sufijos diminutivos.

- Modificación externa: cuantificadores minimizadores, aproximativos o difusores.

- Términos o expresiones más suaves en el contenido significativo.

- Empleo de palabras extranjeras.

(4)

I.: (...) yy luego otra vez / iba con mi hermana/ y yy nos cogieron dos chicas que debían ser drogatillas o algo así/ y entonces se nos puso una cadaa una al lado de mi hermana/ y otra al lado de mí / y entonces nos iban como empujando con el hombro ¿sabes? de forma que yo me chocaba contra mi hermana/ pero aparte por este lado estaba notando el calor de esta chica/ y era un poco desagradable// yy entonces ella estaba diciendo / que le diéramos el dinero/ que le diéramos el tal / y entonces mi hermana/ quee tiene mucho más carácter que yo/ decía que no llevábamos nada

(MADR-VAL_M13_018)

5. Recursos que justifican

- Construcciones justificadoras

- Construcciones de excusa

(5)

E.: claro/menudo

I.: hm

E.: lío ¿no?/ es decir

I.: pee- sí es bastante bastante lío lo que pasa que a lo mejor como eso como era pequeño

E.: claro/

I.: noo parece tanto lío / lo único/ si acaso / también recordaba / hoy dormíaa en el cuarto de mis padres mañana dormía en mi cuarto / o ¿sabes?

(MADR-VAL_H13_013)

6. Recursos que implican al tú en lo que se dice o se hace

- Elipsis de la conclusión, estructuras suspendidas o estructuras truncadas

- Partículas discursivas y expresiones de control de contacto con el interlocutor

- Formas de tratamiento y fórmulas apelativas 
(6)

I.: construido una nueva peroo el cura ha cambiado y no nos dejan

A1.: no / han construido / una residencia

I.: pero detrás está la iglesia

A1.: pero una iglesia chiquitita porque antes era

E.: hm

I.: ¿chiquitita? jtiene mazo de sitio / para nosotros!

A1.: bueno para vosotros sí

I.: pero vamos/ como tampoco soomos muy católicos pues tampoco querraán

E.: ya / uhum / sí bueno porque

I.: que es normal ¿sabes? peroo

(MADR-VAL_H13_014)

7. Recursos que impersonalizan y desfocalizan

- Impersonalizaciones mediante la ocultación de la primera persona en la segunda persona, en un interlocutor general o en el juicio de la mayoría

- Impersonalizaciones a través de estilo directo

- Impersonalizaciones a través de partículas o expresiones de objetivación

(7)

E.: ¿de qué te encargas tú? / deel- ¿de qué?

I.: yo me encargo de los chavalees mayores $(. .$.

E.: ¿has elegidoo? < risas = "E" /> / ¡bueno! // difícil ¿iverdad? ( ...)

I.: se plantean problemas algunas veces quee// dices <cita $>$ joder! si es que estoo ¡no sé si lo sé resolver yo! </cita> (risas)/ pero bueno / la verdad es quee es curioso porque lo de la are- la eh coeducación/ o sea/ es muy interesante porque // eeh // tú estás dando unos valores a- a unas personas/ y a la vez te están transmitiendo a ti y tú estás aprendiendo

(MADR-VAL_H12_008)

Número de mecanismos atenuantes por acto de habla, considerada la unidad de análisis. Se analiza en cada acto la cantidad de mecanismos atenuantes empleados; así, un mayor número de mecanismos en un acto supone un grado más alto de atenuación (Briz, 2007).

(C) Posición sintáctica en la que se encuentra el mecanismo atenuante más significativo de cada acto de habla. Las variantes resultantes de esta variable atienden a si el mecanismo atenuante aparece integrado en el acto de habla, intercalado e interrumpiendo o alterando la unidad sintáctica, intercalado con función rectificadora, en posición inicial que anticipa, en posición final que repara o acota, o en posición inicial y final a la vez. 
(D) A las variables anteriores, puramente lingüísticas, se han de añadir las contextuales. Así, el análisis de la atenuación, ha contado con el cálculo de un conjunto de factores pragmáticos y discursivos que ayudan a reconocer -con un alto porcentaje de garantías- si en un contexto determinado el hablante empleó una forma lingüística con una intención atenuante. Estas variables son las siguientes:

- Carga semántica del contenido del acto de habla. Se analiza si el mensaje emitido afecta o no afecta a la imagen de alguno de los interlocutores o de personas que no se encuentran en el evento del acto comunicativo.

- Tipo de acto de habla en función de su fuerza ilocutiva. Se han identificado los siguientes tipos: directivos en beneficio del hablante o del oyente, asertivos de opinión o de expresión del estado factual de la realidad, compromisivos y expresivos.

Asimismo, las investigaciones sobre atenuación en PRESEEA incluyen otras variables pragmático-discursivas, que permiten controlar factores condicionantes de la variabilidad en circunstancias determinadas: la temática en desarrollo cuando se produce el acto de habla con atenuación (especializada o no especializada), el registro (formal, medio, informal), la modalidad textual (argumentación, exposición, narración y descripción), el propósito funcional predominante (transaccional o interpersonal), el carácter del discurso atenuado (reproducido o no reproducido), la toma de turno (atenuación en turno propio o robado) y el control temático (atenuación en un turno en el que se produce, o no se produce, cambio de tema). Sin embargo, este particular bloque de variables, no serán comentadas aquí por no haber resultado de interés, en general, en los estudios hasta ahora realizados.

Por último, se han considerado las variables sociales de edad, sexo y nivel de instrucción de los informantes, apuntadas previamente y definidas en la metodología sociolingüística de PRESEEA. Este grupo de factores, junto con la variable que puede considerarse dialectal-cultural -aquí las correspondientes a las urbes estudiadas hasta el momento en relación con la estrategia de atenuación- son aquellas que permiten identificar y describir los patrones sociopragmáticos y dialectales como fin fundamental de nuestro trabajo.

\section{Hacia una descripción de patrones sociolectales y dialectales de la atenuación en español}

En la última década, como se ha mencionado previamente, se ha desarrollado el estudio sociolingüístico y dialectal de la atenuación, especialmente en el marco de PRESEEA. El establecimiento de una ficha tan detallada como la establecida para el análisis cualitativo y codificación del fenómeno (Albelda \& Cestero, 2011, Cestero \& Albelda, 2012; Albelda et al., 2014), así como la elaboración de una Guía de estudios de la atenuación (Cestero \& Rodríguez, 2014; Briz \& Albelda, 2013; Albelda et al., 2014), en 
la que se exponen los procedimientos analíticos, ha permitido su estudio en profundidad y, a partir de los resultados, el conocimiento de la existencia de patrones sociopragmáticos. La propuesta de trabajo coordinado que está en la base del macroproyecto, por su parte, ha propiciado la igualdad metodológica y, con ella, la comparabilidad de datos, lo que nos está llevando, como mostraremos en las páginas que siguen, al establecimiento de patrones dialectales.

Como se ha dicho, PRESEEA propone llevar a cabo estudios sociolingüísticos, para los que se atiende a tres factores sociales: la edad, el sexo y el nivel de instrucción de los interlocutores. Por otro lado, uno de los presupuestos básico del PRESEEA es comparar los resultados obtenidos en los diferentes estudios realizados, de manera que la variación diatópica y cultural constituye un objetivo general fundamental. Teniendo en cuenta estos dos grupos de factores y los tres mencionados en el apartado de metodología (tipo de atenuación, mecanismos para atenuar y aspectos pragmáticos y discursivos implicados en los actos de habla con atenuación), se han analizado ya, cualitativa y cuantitativamente, los actos de habla con atenuación de un subcorpus de 18 entrevistas semidirigidas (aproximadamente nueve horas de grabación) de distintas urbes: Madrid (Molina, 2005, 2015; Cestero, 2011a, 2011b, 2012a, 2012b, 2015, 2017), Valencia (Albelda, 2011, 2012, 2013), Las Palmas de Gran Canaria (Samper, 2013, 2017), Granada (Montoro del Arco, 2016)5 y Santiago de Chile (Guerrero, en prensa), así como de un subcorpus de 12 interacciones, correspondientes a sujetos de nivel bajo y alto de instrucción, de Puebla de Zaragoza, en México (Palacios, 2017, 2019, en prensa). Además, se han realizado comparaciones de resultados de las ciudades mencionadas, lo que ha permitido comenzar a establecer patrones sociopragmáticos y geolectales (Cestero \& Albelda, 2012; Cestero, Albelda, Guerrero \& Samper, 2020; Cestero, en prensa). Se trata de estudios sobre atenuación en corpus PRESEEA en los que se atienden todas las variables y variantes en la investigación general del fenómeno, y conforman lo que se considera la modalidad A en PRESEEA_ATENUACIÓN.

En España, Madrid sigue siendo la capital irradiadora de norma y, quizás por ello, una urbe extremadamente sensible a prestigios abiertos y encubiertos y a marcadores sociolingüísticos identitarios. La atenuación, como estrategia comunicativa de cooperación interaccional, que proyecta una imagen, autoprotegiéndola y protegiendo, a su vez, la imagen de los demás, es de uso bastante frecuente, más que en Las Palmas de Gran Canaria, enclave puente entre las zonas hispanohablantes de los dos lados del Atlántico, pero menos que en Valencia, centro bilingüe en el que la comunicación en español presenta como característica importante la minoración de la fuerza ilocutiva de los actos de habla. En el caso de la capital española, la estrategia no es, como hasta ahora se había considerado, un marcador sociolingüístico del habla y la identidad de las mujeres, pues la emplean más los hombres, y a juzgar por la escasa diferencia de la frecuencia de uso, podría ser un patrón sociopragmático 'en cambio' en las capitales 
de las comunidades valenciana y canaria, aunque en ellas aún atenúan más las mujeres que los hombres en registro medio. La mayor consciencia sociolingüística e interaccional de los adultos y las personas instruidas podría explicar, por otro lado, que sea el habla de los sujetos de tales sociolectos la que presente una proporción de atenuación más alta en las tres comunidades, si bien es necesario mencionar la especificidad de Valencia, donde las personas con un nivel de instrucción medio sobrepasan considerablemente en uso de atenuación a las de nivel universitario, así como las de la capital canaria, en la que los jóvenes superan al resto, pues emplean más recursos atenuadores por acto.

Si los hechos que acabamos de mencionar llevan a pensar en una posible convergencia o similitud en patrones sociolingüísticos en frecuencia de uso de la atenuación en las tres urbes estudiadas, la estrategia atenuadora de cada una de ellas muestra diferencias claras que las singularizan y que podrían considerarse marcas identitarias dialectales, esto es, culturales. En la capital española, los recursos de atenuación más asiduos son el empleo de signos paralingüísticos, de marcadores correctores y de formas de modificación externa, la impersonalización mediante ocultación en otro, en un interlocutor general o en el juicio de la mayoría y la expresión de aserciones en forma de duda o de probabilidad. Valencia, sin embargo, suele atenuar utilizando marcadores discursivos que impersonalizan incidiendo en la franqueza de lo dicho y con ello objetivando, justificaciones y excusas y fórmulas fáticas, mediante la expresión de aserciones en forma de duda o de probabilidad y paralenguaje. Por último, en la capital canaria se atenúa habitualmente a través de modificadores externos, con justificaciones y excusas, expresando las aserciones en forma de duda o de probabilidad, con fórmulas fáticas y con una conveniente selección léxica.

Como puede deducirse de los datos apuntados, y a tenor de la categorización funcional de los recursos de atenuación que hemos presentado en el marco teórico, la estrategia atenuadora es diferente en las tres comunidades. En el continuo que los conjuntos estratégicos conforman, de la máxima a la mínima exposición de la imagen, de la atenuación más 'abierta' a la más 'encubierta', más directa a menos directa, la comunidad meridional parece posicionarse en lugares de mediana exposición, con una estrategia atenuadora de minimizar o difuminar la cantidad o cualidad de lo dicho, aunque con un balanceo entre los puntos más equidistantes, pues también es frecuente atenuar justificando; mientras que la capital española se sitúa hacia el polo de máxima exposición, atenuar corrigiendo o reparando su propio acto de habla o parte de él, quizás por ser la urbe irradiadora de norma, y la valenciana hacia el de mínima exposición, desfocalizando y distanciándose de lo hecho y lo dicho, tal vez por tratarse de una comunidad bilingüe y de sujetos que, en ella, hablan en español.

Ahora bien, el análisis sociopragmático de los datos permite vislumbrar estrategias sociolectales que informan de estilos interactivos diferentes de hombres y mujeres, 
jóvenes, adultos y mayores y personas con diferentes niveles de instrucción, y que son los que explican los posicionamientos distintos de la estrategia más general en el continuum de exposición de la imagen. Así, en Madrid, se distancian del patrón de atenuar corrigiendo o reparando lo dicho o lo hecho mediante signos paralingüísticos los hombres, que, como segundo recurso más frecuente, atenúan desfocalizando, los adultos, que también se distancian de lo que dicen o hacen como segundo recurso de atenuación, y las personas con un nivel de instrucción alto que, en este caso, como más atenúan es desfocalizando mediante impersonalizaciones. En Valencia, la variabilidad es mayor que en las otras dos comunidades, aunque los sujetos se mueven habitualmente en el extremo de la desfocalización; las mujeres y los adultos son los que más se distancian de lo que dicen o hacen, por tanto, los que menos se exponen al atenuar, mientras que las personas con un nivel de instrucción alto atenúan mayoritariamente corrigiendo o reparando y los jóvenes rebajan la aserción expresándola en forma de duda o de incertidumbre. En Las Palmas, el patrón general solo se ve alterado por los mayores, que presentan como estrategia más empleada la justificación, y por los adultos, que atenúan desfocalizando como segunda estrategia más usada.

Estos últimos datos, a su vez, se relacionan, en última instancia, con la función de la atenuación en la interacción comunicativa, al menos en la situación semiformal analizada. Sin excepción, por el tipo de actividad comunicativa (entrevista semiformal), la función asidua para la que se atenúa en las tres comunidades tratadas es la de autoprotección, seguida de la de prevención y, casi sin ejemplos, de la de reparación. Pero también en este caso hay una diferencia considerable en las tres urbes en la proporción de atenuación preventiva, que nos permite hablar, de nuevo, de estilos interaccionales diferentes, ahora en atención a lo que podríamos considerar 'proyección hacia el otro', si tenemos en cuenta que la autoprotección proyecta la mayor relevancia discursiva hacia sí mismo, pues coloca como punto central la imagen propia, mientras que la prevención (y reparación) puede considerarse un estilo que proyecta 'al otro', al que otorga mayor importancia en la actividad sociocomunicativa interactiva. Es destacable, en este sentido, que los valencianos, que son los que menos se exponen al atenuar, empleando mayoritariamente estrategias de distanciamiento y desfocalización, sean los que, proporcionalmente, más enfocan su estilo discursivo hacia el otro, ya que es muy pequeña la diferencia que se da en la frecuencia de uso de atenuación para autoprotección y prevención. Lo mismo ocurre en Madrid, aunque, en este caso, la distancia entre las dos funciones de atenuación es mayor. La comunidad canaria, sin embargo, presenta claramente un patrón de discurso con autoprotección, pues la diferencia entre la atenuación para autoprotegerse y prevenir es muy grande, lo que convierte a la primera en usual y a la segunda en peculiar.

En relación con el estilo discursivo-interaccional, parece vislumbrarse, a partir de los resultados obtenidos en la investigación realizada, que las características sociales de 
los hablantes conforman subestilos que son dignos de reseñar y que muestran, de nuevo, identidad sociocultural. En Madrid, los que mayor proyección hacia 'el otro' manifiestan a través de la función de la atenuación, como prevención, son de manera significativa las mujeres, los jóvenes y las personas con nivel medio de instrucción. En Valencia, solo resulta relevante el estilo discursivo proyectado hacia 'el otro' en las personas con nivel medio de instrucción, aunque es destacable la tan frecuente atenuación preventiva de adultos y mayores. Finalmente, en Las Palmas, destacan las personas con nivel bajo y medio de instrucción y los jóvenes y adultos por un mayor uso de atenuación preventiva en su interacción que los sujetos del resto de sociolectos, aunque, como se ha mencionado, en esta comunidad atenuar para prevenir es muy poco frecuente.

Si llevamos nuestra mirada ahora al otro lado del Atlántico, los hallazgos resultan aún más interesantes. Como se ha mencionado con anterioridad, contamos ya con el análisis completo de la atenuación en Santiago de Chile y con el correspondiente a los niveles de instrucción bajo y alto, por tanto los extremos, de Puebla, en México. Tenemos, por tanto, datos de una urbe del sur, capital con variedad normativa reconocida, y de otra centroamericana, de enclave estratégico entre la Ciudad de México, irradiadora de norma, y la zona costera de unión con el continente europeo. Sus enclaves y seguramente la identidad sociocultural explican, creemos, la gran diferencia que existe entre ellas en el uso de atenuación, así como la existencia de patrones similares a los españoles, especialmente a los de Madrid, de ambas comunidades, que podría apuntar movimientos de convergencia, sin obviar las diferencias identitarias culturales de cada comunidad.

En Santiago de Chile, se atenúa bastante más que en Madrid y que en Las Palmas, aunque algo menos que en Valencia. Ahora bien, esa proporción de atenuación es extraordinariamente menor que en la ciudad mexicana, donde, normalizando frecuencias, pues de Puebla solo tenemos datos de los niveles bajo y alto de instrucción, se produce el triple de actos de habla atenuados y se utiliza casi el triple de recursos de atenuación.

Como en la capital española, en las dos comunidades americanas son los hombres, no las mujeres, los que más atenúan y, en ambas, ahora a diferencia de lo que ocurre en las ciudades españolas, la frecuencia de atenuación en interacción disminuye a medida que aumenta la edad; así, son los jóvenes los que más atenúan, seguidos de los adultos y de los mayores, lo que podría apuntar a movimientos de cambios sociopragmáticos en marcha, que no se ven todavía en España. Finalmente, cada urbe americana muestra especificidad en relación con el empleo de atenuación según el nivel de instrucción, pues Santiago, distanciándose del patrón español, presenta mayor empleo de atenuación en el habla de los sujetos con menor instrucción, mientras que, en Puebla, como en Madrid y en Las Palmas, son los sujetos con un nivel de instrucción alto los que más atenúan. 
En el continuum de mayor a menor exposición de imagen que permite caracterizar la estrategia atenuadora que individualiza, y confiere marca identitaria, las comunidades americanas estudiadas hasta ahora, Santiago de Chile y Puebla, ocupan lugares intermedios, aunque especialmente Puebla, se asemeja bastante a la comunidad meridional-puente española. Los recursos más empleados por los chilenos son las justificaciones y excusas, los marcadores correctores, la expresión de aserciones en forma de duda o probabilidad y los movimientos concesivos; ello permite considerar como estrategias habituales en la capital la atenuación justificando, corrigiendo o reparando, rebajando la aserción o realizando concesiones. Por su parte, los hablantes de puebla atenúan más frecuentemente mediante marcadores correctores, impersonalizaciones, modificación morfológica externa, fórmulas fáticas de confirmación y movimientos concesivos, lo que lleva a caracterizarlos por su estrategia atenuadora de corrección y reparación, desfocalización, minimización o difuminación de cantidad o cualidad de lo dicho, implicación del 'otro' y concesiones al 'otro', en un balanceo que se asemeja al de la comunidad canaria española.

No podemos comprobar patrones sociopragmáticos en Puebla, pues no disponemos aún de todos los datos, si bien los resultados de los trabajos de Palacios muestran un patrón similar en la estrategia atenuadora en lo que al nivel de instrucción bajo y alto se refiere, aunque como segundo recurso los hablantes con nivel bajo prefieren la desfocalización mediante impersonalización y los de instrucción alta, la modificación morfológica externa. En Santiago de Chile, los estudios de Guerrero arrojan resultados de gran interés; así, las estrategias atenuadoras de hombres y mujeres son las indicadas como generales, al igual que las de los sujetos de instrucción baja y media, jóvenes y mayores; por tanto, las especificidades estratégicas se encuentran en las personas instruidas, que atenúan mayoritariamente rebajando la aserción, expresándola en forma de duda o de incertidumbre, y en los adultos, que prefieren los mecanismos correctores.

Por último, la función para la que atenúan los hispanohablantes chilenos y americanos se asemeja a los datos encontrados en la comunidad meridional-puente española, y se diferencia, marcando estilos discursivos-interaccionales propios, de lo que acontece en el habla madrileña y valenciana. Las urbes americanas presentan un patrón de discurso con autoprotección claro, pues la diferencia entre la atenuación para autoprotegerse y prevenir es muy grande, más en la ciudad mexicana que en la chilena, lo que lleva a considerarlos estilos discursivos-interaccionales centrados en la proyección de la propia imagen, que se autoprotege continuamente. Ahora bien, podemos hablar, también, de subestilos propios de sociolectos determinados: en Santiago de Chile, los hombres, los adultos y mayores y las personas instruidas atenúan más para prevenir que el resto (especialmente los hombres más que las mujeres, lo que marca una diferencia muy clara con respecto a los patrones madrileños a los que se asemejaban hasta ahora los chilenos). En Puebla, las mujeres atenúan algo 
más que los hombres para prevenir, y los jóvenes más que el resto, pero la atenuación con función preventiva, centrada en 'el otro' es tan inusual que no podemos hablar de característica o estilo sociocomunicativo.

Una vez ofrecidas las descripciones detalladas de los patrones de las zonas estudiadas, veamos como conclusión un sintético resumen de los datos cuantitativos en contraste. Los siguientes gráficos permiten visualizar las diferencias y semejanzas rápida y claramente de la información obtenida sobre patrones sociopragmáticos y geolectales en Madrid, Valencia, Las Palmas de Gran Canaria, Santiago de Chile y Puebla (México) ${ }^{6}$ :

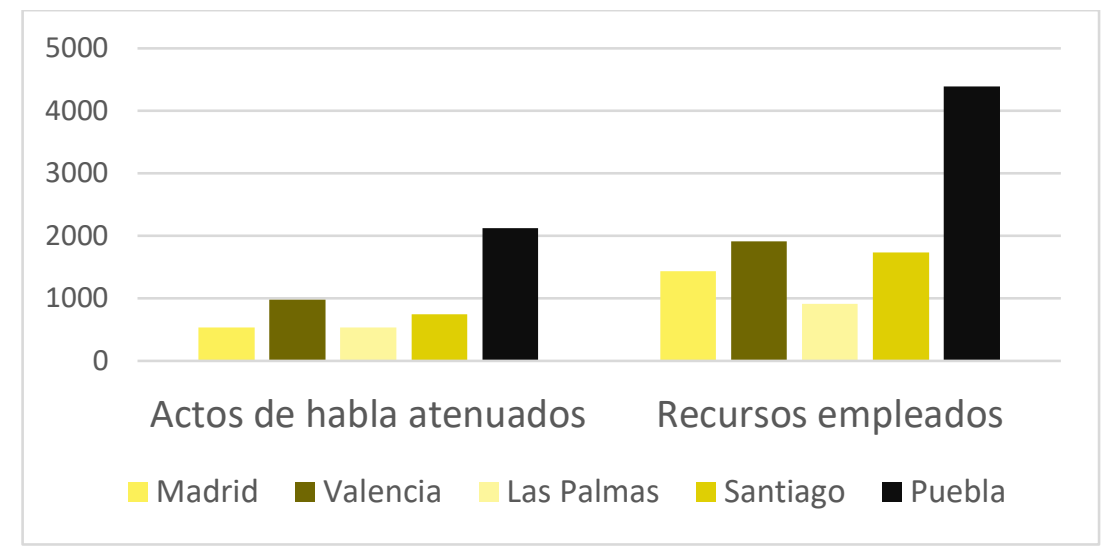

Gráfico 1. Uso de la atenuación en Madrid, Valencia, Las Palmas, Santiago de Chile y Puebla. 


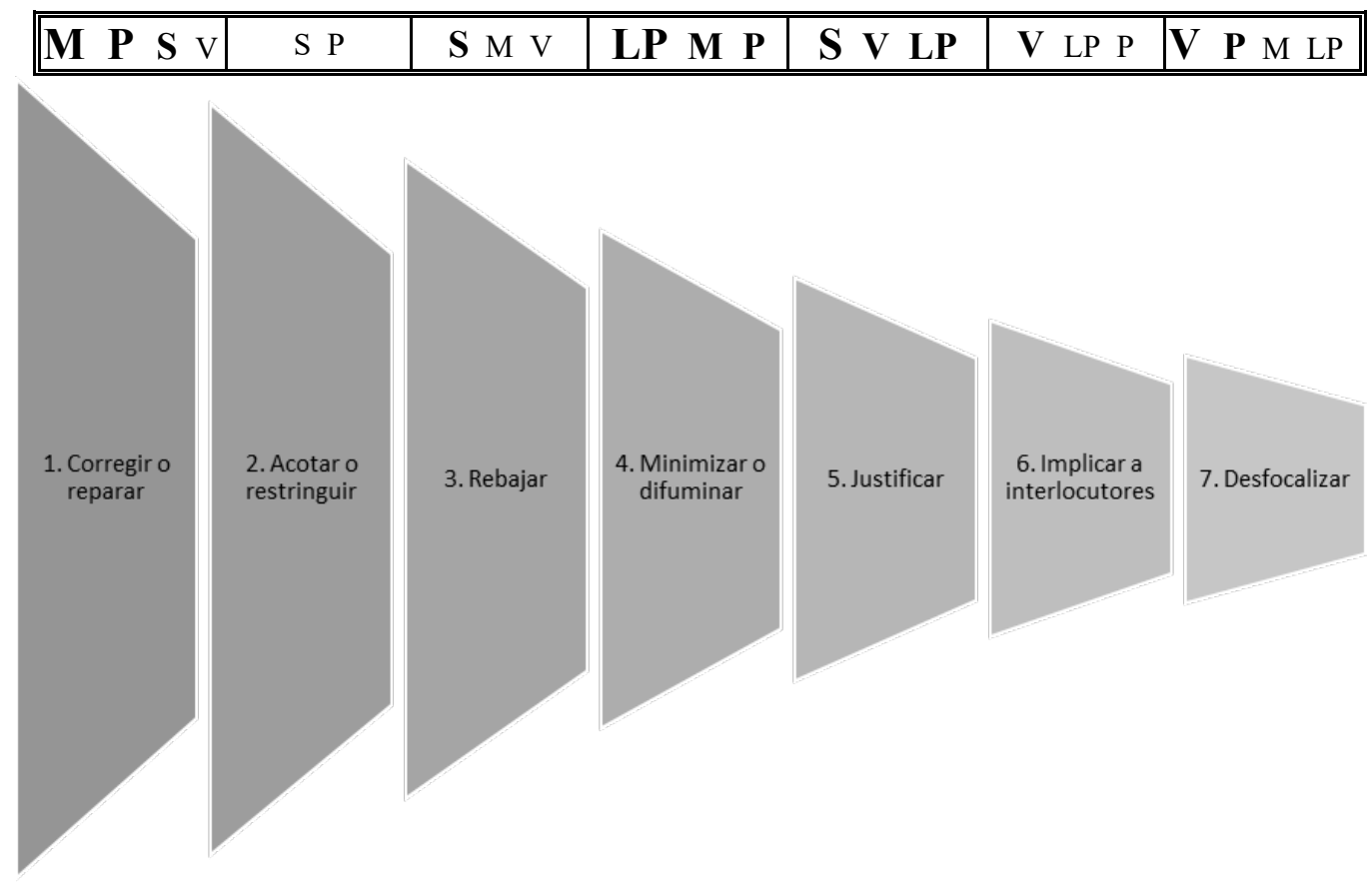

Gráfico 2. Estrategia atenuadora en Madrid, Valencia y Las Palmas, Santiago de Chile y Puebla.

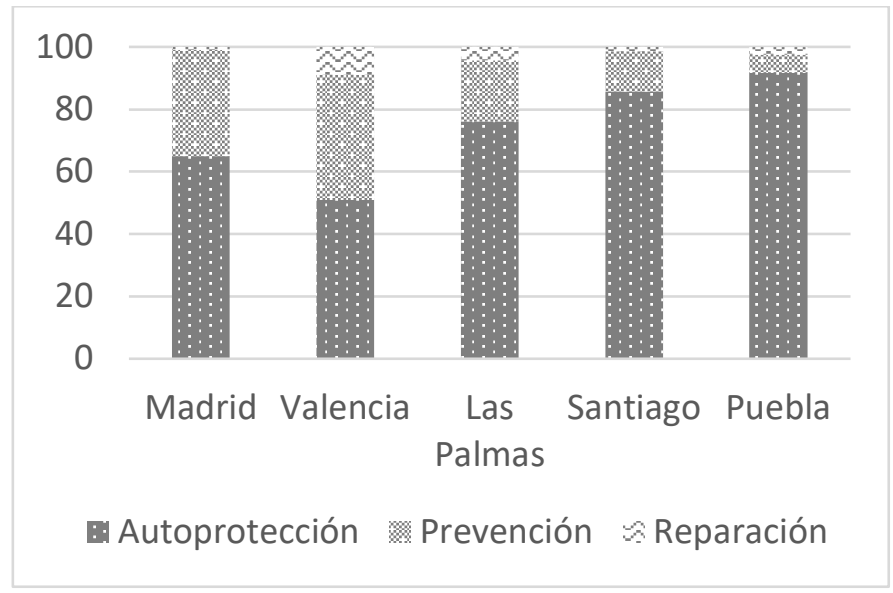

Gráfico 3. Función de la atenuación en Madrid, Valencia y Las Palmas, Santiago de Chile y Puebla.

\section{Perspectivas de futuro en la descripción de patrones sociolectales y dialectales de la atenuación en español}

Como puede apreciarse por lo expuesto hasta aquí, los estudios generales de la atenuación en corpus PRESEEA han proporcionado datos de gran interés, que han permitido comprobar la existencia de patrones sociopragmáticos, por un lado, $\mathrm{y}$ 
geolectales, por otro, que informan sobre estrategias interaccionales que caracterizan y confieren identidad a las distintas comunidades y a los sujetos que pertenecen a ellas. En algunos casos, además, parecen mostrar movimientos de convergencia hacia lo que acontece en la capital española, pero, en otros, son muestra de posibles procesos de cambio o de la existencia de estilos sociocomunicativos.

Asimismo, otra de las conclusiones que se extrae de este estudio variacionista tiene que ver con la validación del método de trabajo, esto es, el diseño de una ficha común y uniforme para el análisis y la codificación de la atenuación y el empleo de un corpus de interacciones del mismo género (entrevista semiformal) y con la misma estratificación sociolingüística en cada zona geográfica. El hecho de que se hayan obtenido tendencias y patrones en cada zona muestra que un método minucioso en el establecimiento y análisis de los parámetros, respetuoso con el contexto común y generoso en el análisis cualitativo es efectivo y válido para la comparación dialectal de una estrategia pragmática como es la atenuación. En ese sentido, la investigación hasta ahora realizada constituye un impulso para la continuación de los análisis de la atenuación en otras áreas geográficas del español.

En la actualidad, ya hay otros trabajos en marcha. Los resultados ofrecidos podrán completarse en breve, cuando se terminen los estudios que atienden al uso general de la atenuación en el habla de Granada, comunidad andaluza española, y Monterrey, enclave serrano en el nordeste de México. Ahora tenemos datos parciales de las dos comunidades, pues para Granada, Béjar (2015) da cuenta de lo que ocurre en 4 entrevistas de sujetos jóvenes y adultos, hombres y mujeres, con nivel de instrucción alto, y Uribe (2017), para Monterrey, en tres entrevistas de mujeres jóvenes, cada una con un nivel de estudio diferente (bajo, medio y alto); no obstante, pueden considerarse ya una valiosa aportación a la configuración sociopragmática y geolectal del fenómeno y por eso nos sirven de colofón de este artículo.

En Granada, al menos en el habla de los sujetos instruidos, según Béjar (2015), de manera semejante a lo que ocurre en la capital española, los hombres atenúan bastante más que las mujeres y los adultos más que los jóvenes. Ahora bien, si en el uso de atenuación la comunidad andaluza parece moverse hacia la convergencia con la urbe irradiadora de norma por excelencia, los recursos de atenuación más empleados por el sociolecto estudiado parecen indicar que los granadinos instruidos se posicionan en los puntos intermedios del continuum de mayor a menor exposición de la imagen, pues en orden, utilizan fórmulas fáticas apelativas de confirmación, expresiones de aserciones en forma de duda o probabilidad, reformulaciones, y modificación interna o externa de lo dicho. Por último, también se diferencian notablemente de los madrileños en la función para la que emplean la atenuación; como en todos los casos, pues no podemos olvidar que se trata de análisis de entrevistas semidirigidas en estilo medio de lengua, los sujetos instruidos de Granada atenúan para autoprotegerse, pero los hombres y los adultos atenúan como prevención en una proporción bastante 
mayor que el resto, lo que permite caracterizar su estrategia como más enfocada "al otro" que la de los miembros del resto de sociolectos.

Finalmente, las mujeres de Monterrey, según Uribe (2017), parecen atenuar frecuentemente en interacción, acercándose al patrón encontrado en Puebla, lo que podría permitir hablar de característica sociocomunicativa mexicana. También como ocurría en Puebla, las que tienen un nivel bajo de instrucción atenúan bastante menos que las que lo tienen medio y alto. Sin embargo, la estrategia atenuadora es algo diferente en este enclave, en el que se emplean mayormente modificadores internos, justificaciones y excusas, fingimiento de incertidumbre o ignorancia, corrección e impersonalización, con lo que se coloca en puntos intermedios del continuum de exposición de la imagen, con estrategias de minimización, justificación, rebaje y corrección de lo dicho o hecho y, en el caso de las mujeres con nivel de instrucción medio, de desfocalización. El estilo interactivo de las mujeres de Monterrey, a juzgar por los datos obtenidos por Uribe (2017) confiere relevancia al actor, que se centra en autoproteger la propia imagen y su proyección, pues es la función que cumple la atenuación casi con exclusividad.

Confiamos en que otros investigadores se animen a emprender el análisis de la atenuación sociopragmática en otros corpus dialectales del español y, de esta manera, podamos seguir construyendo el mapa diatópico de la atenuación.

\section{REFERENCIAS BIBLIOGRÁFICAS}

Albelda, M. (2011). Variación sociolingüística en las estrategias de atenuación del corpus PRESEEA-Valencia del sociolecto alto. En A. M. Cestero, I. Molina \& F. Paredes (Eds.), La lengua, lugar de encuentro. Actas del XVI Congreso Internacional de la ALFAL (pp. 1857-1866). Alcalá de Henares: Servicio de Publicaciones.

Albelda, M. (2012). Estudio sociolingüístico (piloto) de la atenuación en el corpus PRESEEA de Valencia. En E. Ridruejo, T. Solías, N. Mendizábal \& S. Alonso (Eds.), Tradición y Progreso en la Lingüística General (pp. 9-28). Valladolid: Universidad de Valladolid.

Albelda, M. (2013). La atenuación: Tipos y estrategias. En J. R. Gómez Molina (Coord.), El español de Valencia. Estudio sociolingüistico (pp. 315-343). Frankfurt am Main: Peter Lang.

Albelda, M. (2016). Sobre la incidencia de la imagen en la atenuación pragmática. Revista internacional de Lingüistica Iberoamericana, 27, 19-32.

Albelda, M. (2018). Variación sociolingüística de los mecanismos mitigadores: Diferencias de uso en edad y sexo. Cultura, Lenguaje y Representación, 19, 7-29. 
Albelda, M. \& Cestero, A. M. (2011). De nuevo, sobre los procedimientos de atenuación lingüística. Español Actual, 96, 121-155.

Albelda, M. \& Briz, A. (2020). Atenuación e intensificación. Pragmática, 567-590.

Albelda, M. \& Cestero, A. M. (2020). Estudio de variación en el uso de atenuación II: Microanálisis de secuencias discursivas, actos de habla y recursos atenuantes. Revista Signos. Estudios de Lingüistica, 53(104), 962-987.

Albelda, M., Briz, A., Cestero, A. M., Kotwica, D. \& Villalba, C. (2014). Ficha metodológica para el análisis pragmático de la atenuación en corpus discursivos del español. (ES.POR.ATENUACIÓN). Oralia, 17, 7-62.

Albelda, M. \& Estellés, M. (Coords.). Corpus Ameresco [en línea]. Disponible en: http://www.corpusameresco.com.

Bazzanella, C., Caffi, C. \& Sbisá, M. (1991). Scalar dimension of illocutionary force. En I. Zagar (Ed.), Speech acts: Fiction or reality? (pp. 63-76). Ljubljana: IPrA Distribution Center for Yugoslavia.

Béjar, R. (2015). Estudio del fenómeno de la atenuación en la ciudad de Granada (nivel sociocultural alto). Revista Electrónica del Lenguaje, 1, 1-28.

Briz, A. (1995). La atenuación en la conversación coloquial. Una categoría pragmática. En L. Cortés (Ed.), El español coloquial. Actas del I Simposio sobre análisis del discurso oral (pp. 103-122). Almería: Servicio de Publicaciones.

Briz, A. (2003). La estrategia atenuadora en la conversación cotidiana española. En D. Bravo (Ed.), Actas del Primer Coloquio Edice (pp. 17-46). Estocolmo: Universidad de Estocolmo.

Briz, A. (2007). Para un análisis semántico, pragmático y sociopragmático de la cortesía atenuadora en España y América. Lingüistica Española Actual, XIX, 138.

Briz, A. (2012). La (no)atenuación y la (des)cortesía, lo lingüístico y lo social: ¿Son pareja? En J. Escamilla \& G. H. Vega (Eds.), Miradas multidisciplinares a los fenómenos de cortesía y descortesía (pp. 33-75). Barranquilla: Universidad del Atlántico.

Briz, A. \& Grupo Val.Es.Co. (2002). Corpus de conversaciones coloquiales. Madrid: Arco/Libros.

Briz, A. \& Albelda, M. (2013). Una propuesta teórica y metodológica para el análisis de la atenuación lingüística en español y portugués. La base de un proyecto en común (ES.POR.ATENUACIÓN). Onomazéin, 28, 288-319. 
Caffi, C. (1999). On mitigation. Journal of Pragmatics, 31, 881-909.

Caffi, C. (2007). Mitigation. Ámsterdam: Elsevier.

Cestero, A. M. (2011a). Estudio sociolingüístico de la atenuación en el corpus PRESEEA-Madrid. En A. Cestero, I. Molina \& F. Paredes (Comps.), Documentos para el XVI Congreso Internacional de la ALFAL (pp. 1897-1906). Alcalá de Henares: Servicio de Publicaciones.

Cestero, A. M. (2011b). Las estrategias de atenuación: Estudio sociolingüístico. En Actas del IX Congreso Internacional de Lingüistica General (pp. 525-542). Valladolid: Universidad de Valladolid.

Cestero, A. M. (2012a). El proyecto para el estudio sociolingüístico del español de España y América. Español Actual, 98, 227-234.

Cestero, A. M. (2012b). Recursos lingüísticos de atenuación en el habla de Madrid. Estudio sociopragmático. En T. Jiménez, B. López, V. Vázquez \& A. Veiga (Eds.), Cum corde et in nova grammatica (pp. 233-246). Santiago de Compostela: Servicio de Publicaciones de la Universidad de Santiago de Compostela.

Cestero, A. M. (2014). Estudio coordinado de la atenuación en el marco del PRESEEA: Propuesta metodológica. En D. da Hora, J. Lopes \& R. Marques (Org.), Estudos Linguísticos e Filológicos. ANAIS (pp. 1-13). Joao Pessoa: ADALTECH-ALFAL.

Cestero, A. M. (2015). La atenuación lingüística en el habla de Madrid: Un fenómeno sociopragmático variable. En A. M. Cestero, I. Molina \& F. Paredes (Eds.), Patrones sociolingüisticos de Madrid (pp. 365-412). Berna: Peter Lang.

Cestero, A. M. (2017). La atenuación en el habla de Madrid: Patrones sociopragmático. RILCE, Revista de Filología Hispánica, 33(1), 57-86.

Cestero, A. M. (En prensa). Uses and resources of mitigation, in contrast. Spanish in Context, 17(2).

Cestero, A. M. \& Albelda, M. (2012). La atenuación lingüística como fenómeno variable. Oralia, 15, 77-124.

Cestero, A. M. \& Rodríguez, L. (2014). Análisis de la atenuación. Guía de estudio de la atenuación. Versión julio-2008 [en línea]. Disponible en: http://preseea.linguas.net

Cestero, A. M., Albelda, M., Guerrero, S. \& Samper, M. (2020). Variación sociopragmática y geolectal en el uso de atenuación. Lengua y Habla, 24, 1-53.

Channell, J. (Ed.) (1994). Vague language. Oxford: Oxford University Press. 
Cutting, J. (Ed.) (2007). Vague language explored. Basingstoke: Palgrave Macmillan.

Cutting, J. (2015). Dingsbums und so: Beliefs about German vague language. Journal of Pragmatics, 85,108-121.

Czerwionka, L. (2012). Mitigation: The combined effects of imposition and certitude, Journal of Pragmatics, 44, 1163-1182.

Figueras, C. (2018). Atenuación, género discursivo e imagen. Spanish in context, 15(2), 258-280.

Fraser, B. (1980). Conversational mitigation. Journal of Pragmatics, 4, 341-50.

Guerrero, S. (En prensa). La atenuación lingüistica en el corpus PRESEEA de Santiago de Chile.

Hernández, N. (2013). Actividad de imagen. Caracterización y tipología en la interacción comunicativa. Pragmática Sociocultural, 1(2), 1-24.

Holmes, J. (1984). Modifying illocutionary force. Journal of Pragmatics, 8, 345-365.

Lakoff, G. (1972). Hedges: A study in meaning criteria and the logic of fuzzy concepts. En P. Peranteau, J. Levi \& Phares (Eds.), Papers from the $8^{\text {th }}$ Regional Meeting of the Chicago Linguistic Society (pp. 183-228). Chicago: Chicago Linguistic Society.

Llopis, A. (2016). De la indeterminación a la atenuación: De alguna manera/forma/modo. Revista Internacional de Lingüistica Iberoamericana, 27, 105122.

Martinovski, B. (2006). Framework for the analysis of mitigation in courts: Toward a theory of mitigation. Journal of Pragmatics, 38(12), 2065-2086.

Mihatsch, W. (2012). Hedges. En C. A. Chappelle (Ed.), The Encyclopedia of Applied Linguistics (pp. 2457-2462). Oxford: Wiley-Blackwell.

Molina, I. (2005). Estrategias de atenuación en el habla de las mujeres madrileñas (barrio de Salamanca de Madrid). Documentos de Español Actual, 6/7, 89-100.

Molina, I. (2015). Estrategias de atenuación en el barrio de Salamanca de Madrid. En A. M. Cestero, I. Molina \& F. Paredes (Eds.), Patrones sociolingüísticos de Madrid (pp. 349-364). Bern: Peter Lang.

Montoro del Arco, Esteban T. (2016). Tipos y estrategias de atenuación en el corpus oral PRESEEA-Granada. Ponencia presentada en el XII Congreso Internacional de Lingüística General, Universidad de Alcalá, 23 al 25 de mayo de 2016. Alcalá de Henares, España. 
Moreno Fernández, F. (1996). Metodología del 'Proyecto para el estudio sociolingüístico del Español de España y de América’ (PRESEEA). Lingüistica, $8,257-287$.

Moreno Fernández, F. \& Cestero, A. M. (En prensa). El proyecto PRESEEA: Desarrollos analíticos. Verba.

Myre, A. (2009). En plan used as a hedge in Spanish teenage language. En A. B. Stenström \& A. Myre Jørgensen (Eds.), Youngspeak in a multilingual perspective (pp. 95-11). Ámsterdam: John Benjamins.

Overstreet, M. (2011). Vagueness and hedging. En G. Andersen \& K. Aijmer (Eds.), Pragmatics of Society (pp. 293-317). Berlín, De Gruyter.

Palacios, N. (2017). Estrategias y mecanismos de atenuación en PRESEEA-Puebla: Instrucción educativa baja. Anuario de Letras, 5(2), 149-181.

Palacios, N. (2019). Estrategias y mecanismos de atenuación en PRESEEA-Puebla: Instrucción educativa alta. En N. Palacios (Ed.), Voces de la lingüística mexicana contemporánea (pp. 235-270). México: El Colegio de México.

Palacios, N. (En prensa). Funciones de la atenuación y factores situacionales en PRESEEA-Puebla: Instrucción educativa baja y alta. En K. Fascinetto \& K. Cárdenas (Eds.), Tópicos de lingüística. México: Benemérita Universidad Autónoma de Puebla.

PRESEEA (2003). Metodología del Proyecto para el Estudio Sociolingüistico del Español de España y de América (PRESEEA), Versión 2.0. 10-2003 [en línea]. Disponible en: http://preseea.linguas.net/Metodología.aspx

PRESEEA (2008). Marcas y etiquetas minimas obligatorias. Versión 1.0. 31-01-2008 [en línea]. Disponible en: http://preseea.linguas.net/Metodología.aspx

Prince, E., C. Bosk \& J. Frader (1982). On hedging in physician-physician discourse. En J. di Pietro (Ed.), Linguistics and the professions (pp. 83-97). Norwood: Ablex.

Samper, M. (2013). La atenuación lingüística en el español de Las Palmas de Gran Canaria. Lingüistica española actual, 35(2), 325-348.

Samper, M. (2017). Análisis sociolingüístico de la atenuación en el español de Las Palmas de Gran Canaria. En M. Albelda \& W. Mihatsch (Eds.), Atenuación e intensificación en diferentes géneros discursivos (pp. 153-168). Madrid: Iberoamericana/Vervuert.

Sbisà, M. (2001). Illocutionary force and degrees of strength in language use. Journal of Pragmatics, 33, 1791-1814. 
Schneider, S. (2013). Atenuación léxica y sintáctica. Oralia, 16, 335-356.

Schneider, S. (2017). Las dimensiones de la intensificación y de la atenuación. En M. Albelda \& W. Mihatsch (Eds.), Atenuación e intensificación en diferentes géneros discursivos (pp. 23-42). Madrid: Iberoamericana/Vervuert.

Thaler, V. (2012). Mitigation as modification of illocutionary force. Journal of Pragmatics, 44, 907-919.

Uribe, L. M. (2017). Estudio general de la atenuación: tres casos de mujeres jóvenes en el habla de Monterrey-PRESEEA. Tesis de Magíster, TEC, Monterrey, México.

Voghera, M. (2013). The emergence of tipo[-N] in Italian. En A. Giacalone, P. Molinelli \& C. Mauri (Eds.), Synchrony and Diachrony (pp. 283-311). Ámsterdam: John Benjamins.

\section{* AGRADECIMIENTOS}

Este artículo se inscribe dentro de las actividades científicas de los siguientes proyectos de investigación: el proyecto PRESEEA titulado AGENDA 2050. Procesos de variación y cambio espaciales y sociales ( $V A R E S-A G E N D A$ 50; ref. PID2019104982GB-C51), del Ministerio de Ciencia, Innovación y Universidades, el proyecto La población migrante de la comunidad de Madrid: factores lingüisticos, comunicativos, culturales y sociales del proceso de integración y recursos lingüisticos de intervención (IN.MIGRA3-CM; ref. H2019/HUM-5772), financiado por la Comunidad de Madrid y el Fondo Social Europeo, y el proyecto La atenuación pragmática en su variación genérica: géneros discursivos escritos y orales en el español de España y América (FFI2016-75249-P), financiado por el Ministerio de Economía y Competitividad.

\section{NOTAS}

1 Por otro lado, son rasgos que, en realidad, definen otros fenómenos semánticos o pragmático-modales: el lenguaje vago, la modalidad del enunciado y de la enunciación, etc.

2 Para una mayor profundización en el concepto de imagen, véanse Hernández (2013) y Figueras (2018).

3 Las grabaciones se transcriben íntegramente. Su transcripción y almacenamiento se han realizado siguiendo las directrices marcadas por el macroproyecto PRESEEA (PRESEEA, 2003, 2008). 
${ }^{4}$ La ficha actual trabaja con 21 recursos de atenuación, al que se le añade posibilidades de identificación de otros no hallados hasta el momento. La atenuación prosódica no se presenta como recurso independiente, sino que se consideran en conjunción con otro tipo de procedimientos lingüísticos segmentales, por lo que se trata como un mecanismo transversal a toda la comunicación y combinable con cualquiera de los otros.

${ }^{5}$ En el caso de Granada, aún no disponemos de los resultados obtenidos por Montoro del Arco (2016) en la investigación completa sobre atenuación; por ello, no los tenemos en cuenta en este estado de la cuestión.

${ }^{6}$ Los datos de Puebla de Zaragoza se ofrecen normalizados en proporción, ya que, como se ha indicado, solo contamos con los resultados del análisis de 12 entrevistas, no de 18. 